\title{
No Statistically Apparent Difference in Antifungal Effectiveness Observed Among Trimethoprim/ Sulfamethoxazole Plus Clindamycin or Caspofungin, and Trimethoprim/Sulfamethoxazole Monotherapy in HIV-Infected Patients with Moderate to Severe Pneumocystis Pneumonia: Results of an Observational Multicenter Cohort Study
}

Yinqiu Huang $\cdot$ Xiaoqing He $\cdot$ Hui Chen · Vijay Harypursat · Yanqiu Lu $\cdot$ Jing Yuan $\cdot$ Jingmin Nie $\cdot$ Min Liu Jianhua Yu · Yulin Zhang · Zhongsheng Jiang · Yingmei Qin · Lijun Xu · Guoqiang Zhou · Defa Zhang •

Xiaohong Chen · Baisong Zheng • Yaokai Chen

Received: October 25, 2021 / Accepted: December 20, 2021 / Published online: January 20, 2022

(C) The Author(s) 2022

\section{ABSTRACT}

Introduction: Pneumocystis pneumonia is a common opportunistic infection in patients with HIV/AIDS, and is a leading cause of death in this population. Early selection of effective

Yinqiu Huang and Xiaoqing He contributed equally to this work.

Supplementary Information The online version contains supplementary material available at https:// doi.org/10.1007/s40121-021-00586-5.

Y. Huang $\cdot$ X. He · V. Harypursat · Y. Lu · J. Yuan . J. Nie $\cdot$ M. Liu $\cdot$ Y. Chen $(\bowtie)$

Division of Infectious Diseases, Chongqing Public Health Medical Center, 109 Baoyu Road, Shapingba District, Chongqing 400036, China

e-mail: yaokaichen@hotmail.com

Y. Huang · Y. Chen

National Key Laboratory for Infectious Diseases Prevention and Treatment with Traditional Chinese Medicine, Chongqing Public Health Medical

Center, Chongqing, China

H. Chen

School of Biomedical Engineering, Capital Medical University, Beijing, China treatment is therefore critical to reduce mortality. We conducted a clinical trial to compare the effectiveness and safety of three different antifungal treatment regimens in HIV-infected patients with moderate to severe PCP.

Methods: Our study was a multicenter, observational prospective clinical trial. We recruited 320 HIV-infected patients with moderate to

J. Yu

Department of Infectious Diseases, Xixi Hospital of Hangzhou, Zhejiang, China

\section{Y. Zhang}

Division of Infectious Diseases, Beijing Youan Hospital, Capital Medical University, Beijing, China

\section{Z. Jiang}

Division of Infectious Diseases, Liuzhou People's Hospital, Liuzhou, Guangxi, China

Y. Qin

Division of Infectious Diseases, The Fourth People's

Hospital of Nanning, Guangxi, China 
severe PCP, and stratified these subjects into a trimethoprim/sulfamethoxazole (TMP-SMX) monotherapy group, a TMP-SMX plus clindamycin group, and a TMP-SMX plus caspofungin group. Patients were invited to participate in 12 weeks of follow-up. Outcomes included the difference in overall mortality and the proportion of overall positive response to treatment in the three groups at weeks 4 and 12, the difference in treatment duration, and the proportion of adverse events among the three groups during the study period.

Results: The probability of survival not statistically different among three treatment groups. Mortality in the TMP-SMX monotherapy group (group 1) was $15 / 115 \quad(13.04 \%)$ vs. $20 / 83$ $(24.10 \%)$ in the TMP-SMX plus clindamycin group (group 2) vs. 24/107 (22.43\%) in the TMP-SMX plus caspofungin group (group 3 ) at week $12 \quad(p=0.092)$. The overall positive response rate to treatment in the three groups was $24.14 \%, 34.94 \%$, and $38.32 \%$, respectively, at week 4 , and $33.91 \%, 38.55 \%$, and $44.86 \%$, respectively, at week 12 . No significant difference in the overall positive response rate to treatment at either week 4 or week 12 was noted

L. Xu

Division of Infectious Diseases, College of Medicine, The First Affiliated Hospital, Zhejiang University, Zhejiang, China

G. Zhou

Division of Infectious Diseases, The First Hospital of Changsha, Hunan, China

D. Zhang

Department of Infectious Diseases, Tianjin Second

People's Hospital, Nankai University, Tianjin, China $(p=0.061, p=0.246)$. Rates of changes to therapy were $6.50 \%(8 / 123)$ in group $1,3.40 \%$ $(3 / 87)$ in group 2 , and $2.70 \%(3 / 110)$ in group 3 , and did not differ significantly among the three groups $(p=0.376)$. There were also no significant differences in adverse events among the three treatment groups of patients with moderate to severe PCP.

Conclusions: Our results indicate that there are no significant statistical differences among the three studied treatment regimens in terms of antifungal effectiveness in HIV-infected patients with moderate to severe PCP. TMPSMX monotherapy is a convenient, cheap, and effective therapeutic drug regimen to treat HIVinfected patients with moderate to severe PCP, and is an appropriate treatment strategy in resource-limited settings.

Clinical Trial Registration: www. ClinicalTrials.gov, ID: ChiCTR1900021195. Registered on February 1, 2019.

Keywords: Trimethoprim/sulfamethoxazole; Clindamycin; Caspofungin; Moderate to severe PCP; Effectiveness and safety; HIV

X. Chen

Department of Infectious Diseases, The Fourth Affiliated Hospital of Harbin Medical University, Harbin, China

B. Zheng

Institute of Virology and AIDS Research, The First Hospital of Jilin University, Changchun 130021, China 


\section{Key Points}

Why carry out this study?

Pneumocystis pneumonia is a common opportunistic infection in patients with HIV/AIDS, and is a leading cause of death in this population. Early selection of effective treatment is therefore critical to reduce mortality.

Some patients show poor sensitivity to TMP-SMX monotherapy. TMP-SMX may be slow to achieve curative effect, which suggests that this treatment option may be inappropriate for critically ill patients.

Primaquine plus clindamycin has been suggested in the current US Department of Health and Human Services (DHHS) guideline as an alternative treatment for patients who have moderate to severe Pneumocystis pneumonia (PCP). Because primaquine is not available in China owing to the elimination of malaria there, it has become necessary to seek a new therapeutic regimen to replace primaquine.

Caspofungin plus TMP-SMX may be a promising drug combination for use in patients with HIV/PCP. However, clinical experience with use of this combination is currently limited.

The aim of this study was to compare the effectiveness and safety of three different antifungal treatment regimens in HIVinfected patients with moderate to severe PCP.

\section{What was learned from the study?}

TMP-SMX monotherapy as a therapeutic drug regimen to treat HIV-infected patients with moderate to severe PCP is an appropriate treatment strategy in resource-limited settings.

\section{INTRODUCTION}

Pneumocystis pneumonia (PCP) is caused by Pneumocystis jirovecii, which was previously classified as a protozoan, but is currently considered to be a fungus on the basis of nucleic acid and biochemical analysis. PCP is one of the commonest opportunistic infections in patients with HIV/AIDS, often occurring in patients with $\mathrm{CD}^{+}{ }^{+} \mathrm{T}$ cell counts less than 200 cells $/ \mu \mathrm{L}$, and is one of the main causes of hospitalization and death among patients with HIV/AIDS. Some early studies showed that the mortality of HIVinfected patients with severe PCP was 36-50\% in the early antiretroviral therapy (ART) era [1]. A past study showed that in patients with HIV/ PCP who had received ART, mortality was 9.9\%; in those who had not received ART, mortality was $12.0 \%$ [2]. In the modern ART era, the mortality rate in HIV-infected patients with PCP has decreased to approximately $10-12 \%$; however, mortality can rise to up to $84 \%$ in those with moderate to severe disease without any treatment [3]. Therefore, early selection of effective treatment is a priority in these patients.

Trimethoprim/sulfamethoxazole (TMPSMX), also known as co-trimoxazole/Bactrim ${ }^{\circledR}$, is currently recommended as first-line treatment of PCP in HIV-infected patients because of its relatively high overall efficacy and the widespread availability of its oral and parenteral formulations [1]. TMP-SMX can relieve the symptoms of PCP and improve prognosis. However, the efficacy rate of patients on TMPSMX monotherapy administered to HIV-infected patients with moderate to severe PCP is 70-80\%, and some patients show poor sensitivity to TMP-SMX monotherapy [4]. TMP-SMX is considered to only destroy trophoblasts, and has no effect on the tomont stage of the organism [5]. In addition, TMP-SMX is slow to act, requiring approximately 5-8 days to achieve curative effect, which suggests that the use of TMP/SMX may be inappropriate for critically ill patients [6]. Therefore, the quest for more effective novel therapeutic regimens to improve the prognosis of patients with 
moderate to severe PCP is an important and currently relevant issue to be addressed [7].

Clindamycin belongs to the lincosamine class of antibiotics, and can act as an alternative to TMP-SMX in terms of therapeutic success and patient safety [8]. Used together with primaquine, clindamycin has been suggested for patients who have moderate to severe PCP in the current US Department of Health and Human Services (DHHS) guideline [9]. Because primaquine is not available in China owing to the elimination of malaria there, it has become necessary to seek a new therapeutic option to replace it. Not many reports in the literature exist with regards to the combination of TMPSMX with clindamycin [10], even though in clinical practice the efficacy of TMP-SMX plus clindamycin appears to be greater than that of TMP-SMX monotherapy.

In recent years, an increasing number of studies report that caspofungin may be used to treat PCP [11]. However, it is believed at present that caspofungin alone should not be used for the treatment of PCP (especially in moderate to severe PCP), as it is unlikely to completely clear Pneumocystis, and may allow PCP recurrence after drug withdrawal [12]. TMP-SMX exerts its effects on trophic forms of $P$. jirovecii, while caspofungin primarily acts on cystic forms of $P$. jirovecii [13], and the combination of caspofungin and TMP-SMX thus has the potential to inhibit the entire life cycle of the Pneumocystis organism [14]. Case reports have described the clinical success of caspofungin when used in combination with TMP-SMX in treating moderate to severe PCP in immunocompromised hosts [15]. A clinical trial involving caspofungin salvage treatment in HIV-infected patients with PCP has shown an appreciably high success rate (80\%, 8/10 patients) [13]. Jin et al. found that HIV-negative patients with moderate to severe $P$. jirovecii pneumonia had good response to caspofungin combined with TMP-SMX [16]. In further studies, four patients with PCP were successfully treated with caspofungin and TMPSMX [17]. Caspofungin may thus be a promising drug for use in patients with HIV/PCP; however, clinical experience with its use is currently limited.
In our multicenter, prospective observational cohort study of data from 16 hospitals throughout China, we aimed to observe and compare the clinical effectiveness and safety of TMP-SMX monotherapy, TMP-SMX plus clindamycin, and TMP-SMX plus caspofungin for the treatment of moderate to severe PCP (defined by the presence of an arterial oxygen pressure of less than $70 \mathrm{mmHg}$ or an arterialalveolar gradient of more than $35 \mathrm{mmHg}$ in a patient with HIV/PCP) in HIV-infected patients.

\section{METHODS}

\section{Patient Population and Study Design}

The present multicenter, observational cohort study was conducted at 16 hospitals in China, from January 2019 to December 2020. Our study received human research ethics approval (Approval No. 2019-003-02-KY) from the Ethics Committee of the Chongqing Public Health Medical Center, and from the individual institutional ethics committees of each of the other 15 hospitals involved in this study (Table S1 in the supplementary material), and was duly registered at the Chinese Clinical Trial Registry (Registration No. ChiCTR1900021195). The study was conducted in accordance with the tenets of the 1964 Declaration of Helsinki. All enrolled patients provided written informed consent.

\section{Inclusion and Exclusion Criteria}

Patients with confirmed PCP were included in our study if they satisfied the following eligibility criteria: (1) over 18 years old; (2) HIV positive; (3) willing to sign informed consent; (4) moderate-to-severe PCP.

Patients were excluded from this study if they (1) were intolerant of or had severe allergy to any of the therapeutic drugs used; (2) had hemoglobin (HGB) levels less than $60 \mathrm{~g} / \mathrm{L}$, white blood cell counts (WBC) less than $1.0 \times 10^{9} / \mathrm{L}$, neutrophil counts $(\mathrm{N})$ less than $0.5 \times 10^{9} / \mathrm{L}$, platelet counts (PLT) less than $50 \times 10^{9} / \mathrm{L}$, blood amylase (AMS) more than two times the 


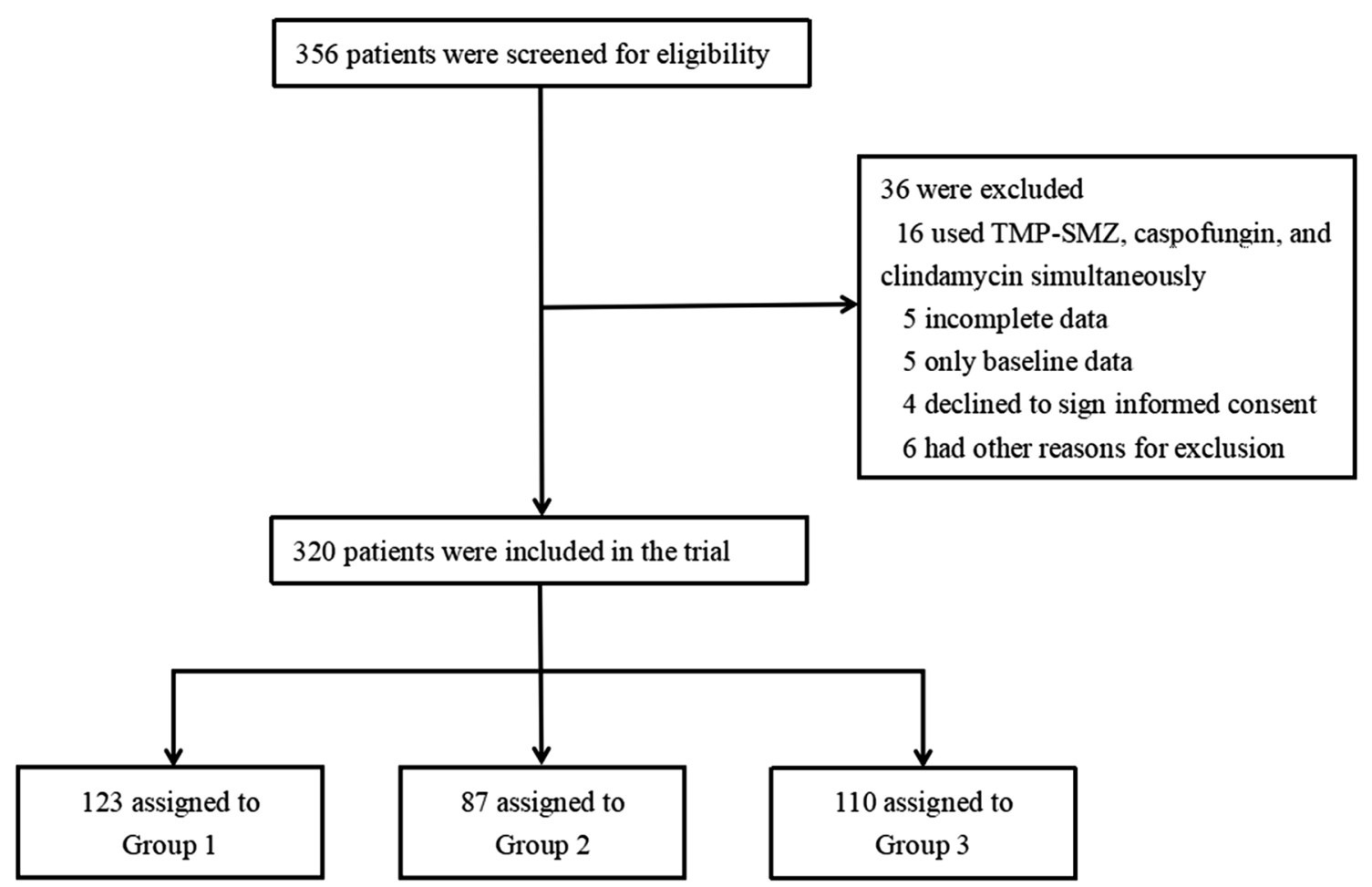

Fig. 1 Flow diagram of the study

upper normal limit (UNL), serum creatinine (Scr) more than 1.5 times UNL, aspartate aminotransferase (AST)/alanine aminotransferase (ALT)/alkaline phosphatase (ALP) more than five times UNL, total bilirubin (TBIL) more than two times UNL, serum creatine phosphokinase (CK) more than two times UNL; (3) had the presence of other serious disease that could have affected the accurate evaluation of efficacy and prognosis; (4) were pregnant or breastfeeding women; (5) had severe mental health illnesses, or used intravenous recreational drugs, (6) had non-Chinese nationality; (7) had mild PCP; or (7) withheld informed consent.

\section{Definitions}

Moderate to severe of PCP was defined as: (1) partial arterial oxygen pressure $\left(\mathrm{PaO}_{2}\right) \leq 70$ $\mathrm{mmHg}$, or whilebreathing room air, an alveolararterial oxygen difference of $(\mathrm{A}-\mathrm{aDO} 2) \geq 35$
mmHg; (2) the presence ofrelevant pulmonary symptoms, including dry cough, shortness of breath, progressive dyspnea, and purpura, and may also have fever; (3) pulmonary infiltrations indicated by chest radiography or computedtomography (CT) performed when PCP was clinically suspected following hospitalization; (4) microbiologicalconfirmation by positive PCR and/or Grocott's methenamine silver (GMS) results for sputum, pulmonaryaspirate, or broncho-alveolar lavage fluid (BALF) samples. If the subject met criteria (1), and (2), and (3), and/ or (4), moderate to severe of PCP was assumed to be present. $\mathrm{PaO} 2$ was measured directly via bloodgas analysis of arterial blood.

Overall positive response rate was defined as having fewer clinical symptoms, improved $\mathrm{PaO}_{2}$, and resolutionof evidence of pneumonitis on chest imaging after treatment.

Treatment failure was defined as persistent fever and worsening hypoxia, and/or radiographic deterioration. 
Table 1 Baseline characteristics of the enrolled patients

\begin{tabular}{|c|c|c|c|c|}
\hline & Group $1(n=123)$ & Group $2(n=87)$ & Group $3(n=110)$ & $p$ value \\
\hline Age, mean years $\pm S D$ & $48.34 \pm 13.31$ & $47.45 \pm 12.80$ & $46.47 \pm 12.61$ & 0.546 \\
\hline Male gender, $n(\%)$ & $100(81.3)$ & $62(71.3)$ & $88(80.0)$ & 0.187 \\
\hline BMI, median kg/m² (IQR) & $20.4(18.5,22.7)$ & $19.6(18.0,21.9)$ & $20.7(18.7,22.9)$ & 0.096 \\
\hline Route of infection, $n(\%)$ & & & & $<0.001$ \\
\hline MSM & $22(17.9)$ & $10(11.5)$ & $16(14.6)$ & \\
\hline Heterosexual & $72(58.5)$ & $63(72.4)$ & $45(40.9)$ & \\
\hline Other & $2(1.6)$ & $1(1.1)$ & $2(1.8)$ & \\
\hline Unknown & $27(22.0)$ & $13(15.0)$ & $47(42.7)$ & \\
\hline ART, $n(\%)$ & $6(4.9)$ & $5(5.7)$ & $3(2.7)$ & 0.574 \\
\hline Smoking, $n(\%)$ & $55(44.7)$ & $25(28.7)$ & $34(30.9)$ & 0.026 \\
\hline Alcohol use, $n(\%)$ & $32(26.0)$ & $11(12.6)$ & $24(21.8)$ & 0.061 \\
\hline \multicolumn{5}{|l|}{ Other medical diseases, $n(\%)$} \\
\hline Diabetes mellitus & $4(3.3)$ & 0 & $6(5.5)$ & 0.067 \\
\hline Hypertension & $2(1.6)$ & 0 & $3(2.7)$ & 0.381 \\
\hline Tuberculosis & $14(11.4)$ & $6(6.9)$ & $14(12.7)$ & 0.395 \\
\hline HIV RNA, median $\log _{10}$ copies/mL (IQR) & $5.7(5.0,6.0)$ & $5.7(5.2,5.9)$ & $5.4(5.0,5.9)$ & 0.518 \\
\hline \multicolumn{5}{|l|}{$\mathrm{CD}^{+}{ }^{+} \mathrm{T}$ cell counts, cells $/ \mathrm{mm}^{3}, n(\%)$} \\
\hline$<50$ & $83(70.3)$ & $72(86.7)$ & $84(79.2)$ & 0.076 \\
\hline $50-100$ & $24(20.3)$ & $7(8.4)$ & $12(11.3)$ & \\
\hline $101-200$ & $9(7.6)$ & $4(4.8)$ & $6(5.7)$ & \\
\hline$>200$ & $2(1.7)$ & $0(0)$ & $4(3.8)$ & \\
\hline $\mathrm{HGB}$, mean $\times 10^{9} / \mathrm{L} \pm \mathrm{SD}$ & $114.55 \pm 18.84$ & $117.01 \pm 18.15$ & $120.27 \pm 21.24$ & 0.084 \\
\hline PLT, median $\times 10^{9} / \mathrm{L}(\mathrm{IQR})$ & $227.0(166.0,286.0)$ & $244.0(172.0,302.0)$ & $246.0(174.5,323.0)$ & 0.166 \\
\hline G test, IQR & $187.0(75.4,450.0)$ & $232.0(70.9,422.9)$ & $202.2(72.3,598.3)$ & 0.702 \\
\hline Scr, median $\mu \mathrm{mol} / \mathrm{L}(\mathrm{IQR})$ & $65.2(53.0,73.5)$ & $62.1(54.7,74.1)$ & $64.5(51.0,78.0)$ & 0.895 \\
\hline AST, median U/L (IQR) & $39.0(26.0,58.0)$ & $36.0(26.0,49.0)$ & $37.5(28.0,51.0)$ & 0.466 \\
\hline ALT, median U/L (IQR) & $21.0(14.0,37.8)$ & $19.0(13.0,37.0)$ & $24.0(14.0,40.3)$ & 0.250 \\
\hline LDH, median U/L (IQR) & $416.5(297.4,502.2)$ & $423.2(335.8,551.0)$ & $446.0(341.6,576.8)$ & 0.105 \\
\hline $\mathrm{PaO}_{2}$, median $\mathrm{kPa}(\mathrm{IQR})$ & $56.0(50.0,62.0)$ & $58.0(52.0,65.0)$ & $57.5(49.8,62.0)$ & 0.236 \\
\hline
\end{tabular}

Data are presented as $n(\%)$, mean $( \pm S D)$, or median (IQR), unless otherwise specified. Group 1, TMP-SMX monotherapy group; group 2, TMP-SMX plus clindamycin group; group 3, TMP-SMX plus caspofungin group

$B M I$ body mass index, MSM men who have sex with men, $A R T$ antiretroviral therapy, $H G B$ hemoglobin, PLT platelets, $G$ test $\beta$-(1,3)-D-glucan test results, $A S T$ aspartate aminotransferase, $A L T$ alanine aminotransferase, $L D H$ lactate dehydrogenase, $\mathrm{PaO}_{2}$ partial arterial oxygen pressure, $\mathrm{Scr}$ serum creatinine 


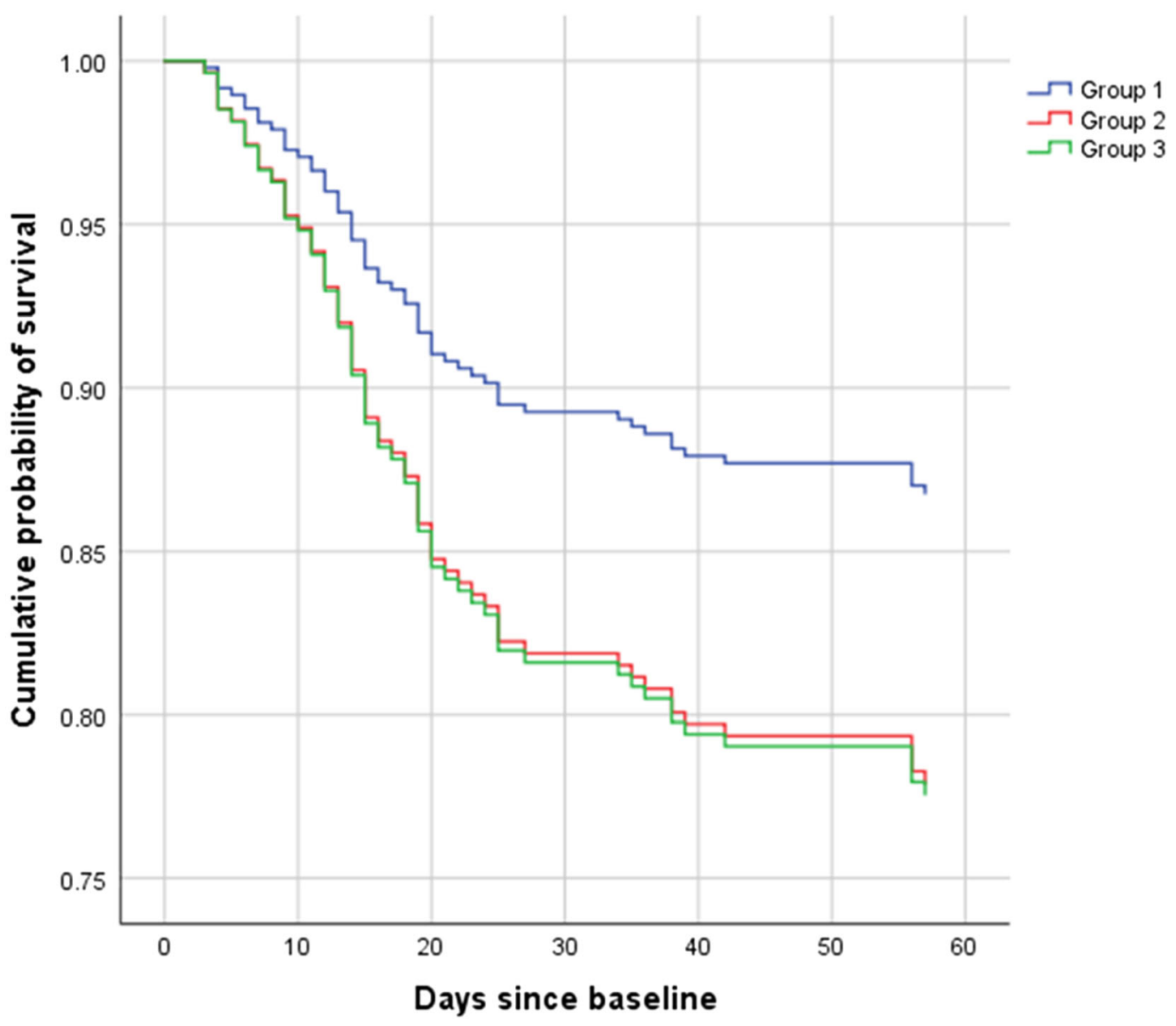

Fig. 2 Overall survival among all 320 participants for three groups. Group 1, TMP-SMX monotherapy group; group 2, TMP-SMX plus clindamycin group; group 3, TMP-SMX plus caspofungin group

\section{Data Collection and Quality Assurance}

Based on a standard operating procedure (SOP) manual, investigators were trained to ensure patientadherence to the study protocol. Data including clinical characteristics, efficacy, safety, and adverse effectswere collected at each follow-up visit during the follow-up period. All raw data were recorded in case reportforms (CRFs) and then transferred to an electronic database via the Medical Research Platform. Withdrawalsfrom the study, or missed visits were fully explained on CRFs. The study monitor reviewed the completedCRFs quarterly to ensure accuracy and diligence of the application of inclusion, exclusion, and withdrawalcriteria, as well as to ensure that information on the CRFs were consistent with those in the source electronicmedical records.

\section{Treatment and Outcomes}

All eligible patients were divided into three groups: Group 1, receiving TMP-SMX monotherapy, Group 2,receiving TMP-SMX plus clindamycin, and Group 3, receiving TMPSMX plus caspofungin. During the studyperiod, we used similar protocols for drug dosage and administration as recommended by internationalguidelines. TMP-SMX was given at a daily dose of $15-20 \mathrm{mg} / \mathrm{kg}$ of trimethoprim and 75-100 $\mathrm{mg} / \mathrm{kg}$ ofsulfamethoxazole. 
Table 2 All-cause mortality rates of the study participants

\begin{tabular}{|c|c|c|c|c|c|c|c|}
\hline & \multicolumn{2}{|c|}{ Group $1(n=123)$} & \multicolumn{2}{|c|}{ Group $2(n=87)$} & \multicolumn{2}{|c|}{ Group $3(n=110)$} & \multirow[t]{2}{*}{$p$ value } \\
\hline & $\begin{array}{l}\text { Total } \\
\text { numbers }(n)\end{array}$ & $\begin{array}{l}\text { Total mortality } \\
\text { (\%) }\end{array}$ & $\begin{array}{l}\text { Total } \\
\text { numbers }(n)\end{array}$ & $\begin{array}{l}\text { Total mortality } \\
\text { (\%) }\end{array}$ & $\begin{array}{l}\text { Total } \\
\text { numbers }(n)\end{array}$ & $\begin{array}{l}\text { Total mortality } \\
\text { (\%) }\end{array}$ & \\
\hline Week 1 & 1 & 0.83 & 4 & 4.60 & 2 & 1.85 & 0.211 \\
\hline Week 2 & 6 & 5.12 & 8 & 9.20 & 10 & 9.26 & 0.420 \\
\hline Week 3 & 11 & 9.48 & 12 & 13.79 & 18 & 16.82 & 0.266 \\
\hline Week 4 & 12 & 10.34 & 15 & 18.07 & 21 & 19.63 & 0.128 \\
\hline Week 12 & 15 & 13.04 & 20 & 24.10 & 24 & 22.43 & 0.092 \\
\hline
\end{tabular}

Group 1, TMP-SMX monotherapy group; group 2, TMP-SMX plus clindamycin group; group 3, TMP-SMX plus caspofungin group

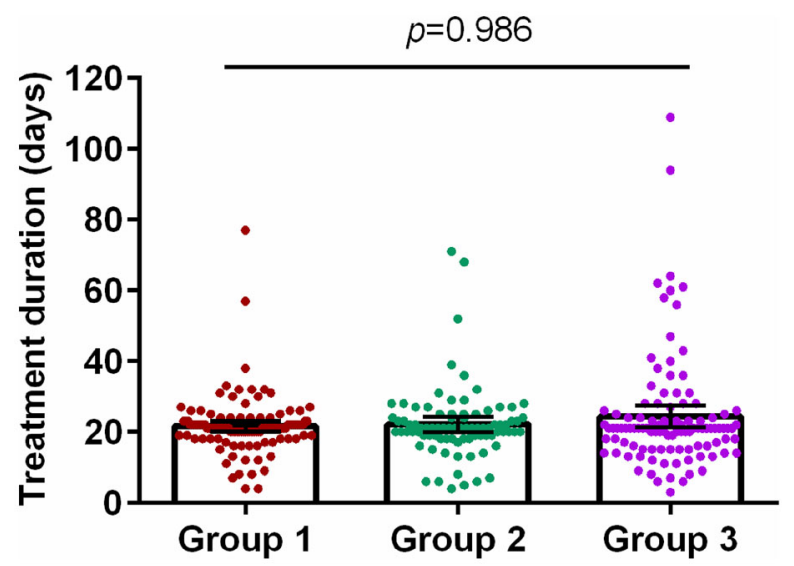

Fig. 3 Duration of moderate to severe PCP treatment. Group 1, TMP-SMX monotherapy group; group 2, TMPSMX plus clindamycin group; group 3, TMP-SMX plus caspofungin group

Clindamycin was given by intravenous injection at dose of $0.6 \mathrm{~g}$ every day. Patientsreceived $70 \mathrm{mg}$ caspofungin intravenously on the first day, and were then subsequently administered a doseof $50 \mathrm{mg} /$ day. All treatment regimens lasted for 3-4 weeks. Adjunctive corticosteroids at the doserecommended by guidelines were administered to all patients. Patients received additional nasal cannulaoxygen therapy, and non-steroidal anti-inflammatory drugs (NSAIDs) as required, as well as oral orintravenous rehydration, electrolyte correction, antipyretics, analgesics, and antiemetic drugs as theirindividual clinical conditions demanded.

The primary outcome was the difference in overall mortality in the three groups at week 4 and week 12 .Secondary outcomes included the proportion of overall positive response to treatment of moderate to severePCP in each group at week 4 and week 12, the differences among the three groups in the duration oftreatment, and the difference in rates of adverse events among the three groups during the study period.

\section{Study Procedures}

After HIV/PCP patients were admitted to hospital, we selected those patients whom we assessed may beeligible for our study, and sought their consent for eligibility screening. After they provided their informedconsent, we assessed their eligibility for study inclusion, and only included into our study those who met ourinclusion criteria but who also did not meet the exclusion criteria of our study. Study visits occurred at week 1 , week 2 , week 3 , week 4 , and week 12 after initiation of PCP treatment. Safety was assessed byinterrogation of the participants for potential development of clinical symptoms, and physical examination forclinical signs, clinical laboratory tests, and documentation of adverse events. Treatment adherence wasassessed by review of clinical diaries that were filled out by medical staff. 
Table 3 Positive response rates in each treatment group

\begin{tabular}{|c|c|c|c|c|c|c|c|}
\hline & \multicolumn{2}{|c|}{ Group $1(n=123)$} & \multicolumn{2}{|c|}{ Group $2(n=87)$} & \multicolumn{2}{|c|}{ Group $3(n=110)$} & \multirow[t]{2}{*}{$p$ value } \\
\hline & $\begin{array}{l}\text { Total } \\
\text { numbers } \\
(n)\end{array}$ & $\begin{array}{l}\text { Positive } \\
\text { response rate } \\
(\%)\end{array}$ & $\begin{array}{l}\text { Total } \\
\text { numbers } \\
(n)\end{array}$ & $\begin{array}{l}\text { Positive } \\
\text { response rate } \\
(\%)\end{array}$ & $\begin{array}{l}\text { Total } \\
\text { numbers } \\
(n)\end{array}$ & $\begin{array}{l}\text { Positive } \\
\text { response rate } \\
(\%)\end{array}$ & \\
\hline Week 1 & 5 & 4.17 & 2 & 2.30 & 3 & 2.78 & 0.781 \\
\hline Week 2 & 17 & 14.53 & 11 & 12.64 & 13 & 12.04 & 0.847 \\
\hline Week 3 & 26 & 22.41 & 24 & 27.59 & 28 & 26.17 & 0.672 \\
\hline Week 4 & 28 & 24.14 & 29 & 34.94 & 41 & 38.32 & 0.061 \\
\hline Week 12 & 39 & 33.91 & 32 & 38.55 & 48 & 44.86 & 0.246 \\
\hline
\end{tabular}

Group 1, TMP-SMX monotherapy group; group 2, TMP-SMX plus clindamycin group; group 3, TMP-SMX plus caspofungin group

Table 4 Treatment for moderate to severe PCP and reasons for switching treatment

\begin{tabular}{|c|c|c|c|c|c|c|c|}
\hline & \multicolumn{2}{|c|}{ Group $1(n=123)$} & \multicolumn{2}{|c|}{ Group $2(n=87)$} & \multicolumn{2}{|c|}{ Group $3(n=110)$} & \multirow{2}{*}{$p$ value } \\
\hline & $\begin{array}{l}\text { Total numbers } \\
(n)\end{array}$ & $\begin{array}{l}\text { Rate } \\
(\%)\end{array}$ & $\begin{array}{l}\text { Total numbers } \\
(n)\end{array}$ & $\begin{array}{l}\text { Rate } \\
(\%)\end{array}$ & $\begin{array}{l}\text { Total numbers } \\
(n)\end{array}$ & $\begin{array}{l}\text { Rate } \\
(\%)\end{array}$ & \\
\hline Total & 8 & 6.50 & 3 & 3.40 & 3 & 2.70 & 0.376 \\
\hline Failure switch & 5 & 4.10 & 1 & 1.10 & 3 & 2.70 & 0.531 \\
\hline $\begin{array}{r}\text { Toxicity } \\
\text { switch }\end{array}$ & 3 & 2.40 & 2 & 2.30 & 0 & 0.00 & 0.268 \\
\hline
\end{tabular}

Group 1, TMP-SMX monotherapy group; group 2, TMP-SMX plus clindamycin group; group 3, TMP-SMX plus caspofungin group

\section{Statistics}

Continuous variables were described as mean $( \pm$ SD) for normally distributed data, or median with interquartileranges (IQR) for non-normally distributed data. Categorical variables were expressed as frequencyrates and percentages. Kolmogorov-Smirnov tests and ANOVA (analysis of variance) tests were used forcontinuous variables. Chi-squared tests, continuity corrections, and Fisher's exact tests were used to teststatistical significance for categorical data. Cox proportional-hazards model were used to analyze andcompare survival rates. All statistical analyses were performed using Statistical Package for the SocialSciences (SPSS) software, version 25.0 software (IBM SPSS, Armonk, New
York, USA), using a statisticalsignificance threshold of $p<0.05$.

Sample size was calculated by using the PASS (Power Analysis and Sample Size) software, version 15 (NCSS,LLC, Kaysville, Utah, USA), according to the following assumptions: a 12 -week mortality rate of $30 \%, 15 \%$, and $10 \%$ in the TMP-SMX monotherapy group, the TMPSMX plus clindamycin group, and the TMPSMX pluscaspofungin group, respectively, with an overall two-sided alpha level of 0.05 , and a statistical power of $90 \%$. Thus, a sample size of 263 patients was required for our study. Considering a $10 \%$ withdrawal rate,we planned to enroll 300 participants, and thus have at least 270 participants for analysis. 
Table 5 Cumulative adverse events of regimens at weeks 4 and 12

\begin{tabular}{|c|c|c|c|c|c|c|c|c|}
\hline & \multicolumn{4}{|c|}{ Cumulative adverse events at week 4} & \multicolumn{4}{|c|}{ Cumulative adverse events at week 12} \\
\hline & $\begin{array}{l}\text { Group 1 } \\
(n=123)\end{array}$ & $\begin{array}{l}\text { Group } 2 \\
(n=87)\end{array}$ & $\begin{array}{l}\text { Group } 3 \\
(n=110)\end{array}$ & $p$ value & $\begin{array}{l}\text { Group 1 } \\
(n=123)\end{array}$ & $\begin{array}{l}\text { Group } 2 \\
(n=87)\end{array}$ & $\begin{array}{l}\text { Group } 3 \\
(n=110)\end{array}$ & $p$ value \\
\hline Skin rash, $n(\%)$ & $5(4.1)$ & $3(3.4)$ & $2(1.8)$ & 0.611 & $5(4.1)$ & $3(3.4)$ & $2(1.8)$ & 0.611 \\
\hline $\begin{array}{l}\text { Gastrointestinal } \\
\text { symptoms, } n(\%)\end{array}$ & $2(1.6)$ & $1(1.1)$ & $0(0)$ & 0.494 & $2(1.6)$ & $1(1.1)$ & $0(0)$ & 0.494 \\
\hline $\begin{array}{l}\text { Mental aberration, } \\
n(\%)\end{array}$ & $1(0.8)$ & $0(0)$ & $0(0)$ & 1.000 & $1(0.8)$ & $0(0)$ & $0(0)$ & 1.000 \\
\hline $\begin{array}{l}\text { Marrow } \\
\text { suppression, } \\
n(\%)\end{array}$ & $49(39.8)$ & $35(40.2)$ & $44(40)$ & 0.998 & $55(44.7)$ & $38(43.7)$ & $49(44.5)$ & 0.988 \\
\hline $\begin{array}{l}\text { Renal dysfunction, } \\
n(\%)\end{array}$ & $6(4.9)$ & $5(5.7)$ & $8(7.3)$ & 0.739 & $6(4.9)$ & $6(6.9)$ & $9(8.2)$ & 0.590 \\
\hline $\begin{array}{l}\text { Hepatic } \\
\text { dysfunction, } \\
n(\%)\end{array}$ & $35(28.5)$ & $23(26.4)$ & $35(31.8)$ & 0.698 & $45(36.6)$ & $30(34.5)$ & $41(37.3)$ & 0.917 \\
\hline $\begin{array}{l}\text { Electrolyte } \\
\text { disturbance, } \\
n(\%)\end{array}$ & $41(33.3)$ & $40(46.0)$ & $39(35.5)$ & 0.151 & $47(38.2)$ & $41(47.1)$ & $41(37.3)$ & 0.312 \\
\hline $\begin{array}{r}\text { Overall adverse } \\
\text { events, } n(\%)\end{array}$ & $89(72.4)$ & $62(71.3)$ & $84(76.4)$ & 0.681 & $94(76.4)$ & $67(77.0)$ & $87(79.1)$ & 0.881 \\
\hline
\end{tabular}

Group 1, TMP-SMX monotherapy group; group 2, TMP-SMX plus clindamycin group; group 3, TMP-SMX plus caspofungin group

\section{RESULTS}

\section{Baseline Characteristics}

During the study period, 356 HIV-infected patients with moderate to severe PCP were screened for eligibility, of whom 320 patients were assigned to an intervention group: 123 patients were assigned to group 1 (the TMPSMX monotherapy group), 87 patients were assigned to group 2 (the TMP-SMX plus clindamycin group), 110 patients were assigned to group 3 (the TMP-SMX plus caspofungin group) (Fig. 1).

The mean age of the cohort was 47.46 years old, and $250(78.13 \%)$ of the 320 patients were men. Most did not have previous exposure to antiretroviral therapy $(95.63 \%, 306 / 320)$, $35.63 \%(114 / 320)$ of the patients had a history of smoking, and $20.94 \%(67 / 320)$ had a history of alcohol use. The most common comorbid diseases present in our cohort were tuberculosis $(10.63 \%, 34 / 320)$, diabetes mellitus (3.13\%, $10 / 320)$, and hypertension $(1.56 \%, 5 / 320)$. Because of the presence of hypoxia and dyspnea in moderate to severe PCP, it was technically difficult and potentially hazardous to carry out bronchoscopic examination in most patients. Approximately $10 \%(32 / 320)$ of our patients underwent bronchoscopic examination and bronchoalveolar lavage for definitive PCP diagnosis, comprising 14 patients in group 1,10 patients in group 2 , and 8 patients in group 3. 
Table 1 shows the clinical characteristics of patients in groups 1-3. There were no significant differences in age, sex, BMI, HIV RNA levels, G test, ALT levels, AST levels, $\mathrm{PaO}_{2}$ levels, HGB levels, and LDH levels among the three groups. Compared with patients in groups 2 and 3 , more patients in group 1 were smokers. The clinical symptoms of PCP on admission included fever, dyspnea, and cough. All patients underwent chest CT scans, and the most common radiological finding was bilateral diffuse ground glass opacification or bilateral predominant consolidation.

\section{All-Cause Mortality}

Adjusted for route of infection and smoking, no significant differences in the 12-week time distribution of survival were found in group 2 $(p=0.093$, HR $1.760,95 \%$ CI $0.911,3.403)$ and group 3 ( $p=0.074$, HR 1.791, 95\% CI 0.945 , 3.392), compared with group 1 (Fig. 2). Among all 320 patients investigated in the study period, group 1 exhibited an all-cause mortality rate of $13.04 \%(15 / 115)$, group 2 exhibited an all-cause mortality rate of $24.10 \%(20 / 83)$, while group 3 exhibited an all-cause mortality rate of $22.43 \%$ (24/107). However, these apparent differences in all-cause mortality at week 12 were not statistically significant ( $p=0.092$; Table 2$)$.

\section{Positive Response Rates}

The duration of anti-PCP treatment was 21 days in each of the three groups. No statistically significant difference in treatment duration was observed among the three groups, and between any two of the three groups ( $p=0.986)$ (Fig. 3).

The overall positive response rate to PCP treatment was $24.14 \%(28 / 116), 34.94 \%$ (29/ $83)$, and $38.32 \%(41 / 107)$ in group 1 , group 2 , and group 3 , respectively, at week $4(p=0.061)$. The overall positive response rate to PCP treatment did not differ significantly among the three groups. The positive response rates of the three groups at week 12 were $33.91 \%(39 / 115)$, $38.55 \%(32 / 83)$, and $44.86 \%(48 / 107)$, respectively ( $p=0.246)$. However, no statistically significant difference in the overall positive response rate to PCP treatment was observed among the three groups (Table 3 ).

\section{Safety Evaluation}

Changes to drug regimens were classified as either caused by adverse effects or caused by suspected failure of treatment. Of 14 of 320 $(4.38 \%)$ patients where a specific therapeutic drug was changed to another drug, 5 (35.71\%) regimens were changed because of adverse effects and 9 (64.29\%) were changed secondary to treatment failure. Drug changes occurred more frequently in group 1 (6.50\%), compared with group $2(3.40 \%)$ and group $3(2.70 \%)$; however, the rates of drug changes were not significantly different among the three groups (Table 4). The median time to change was 12 days in group 1,24 days in group 2 , and 7 days in group 3 , regardless of the reason for change.

Among the three treatment groups, adverse effects mainly presented as bone marrow suppression ( $p=0.998, p=0.988)$ and electrolyte disturbances $(p=0.151, p=0.312)$, and there was no significant difference among the three groups in the occurrence of any adverse event at both week 4 or 12 . Three patients had gastrointestinal symptoms (two patients in group 1 , one patient in group 2 , and none in the group 3), with no statistical difference in the occurrence of gastrointestinal symptoms among the three groups at both week $4(p=0.494)$ or week $12(p=0.494)$. In addition, only one patient appeared to develop central nervous system symptoms (in a patient in the TMP-SMX monotherapy group) in our study. Furthermore, we found no statistically significant differences in the rates of skin rash $(p=0.611)$ and renal dysfunction $(p=0.590)$ among three groups at week 12 (Table 5).

\section{DISCUSSION}

Currently, the first choice of therapeutic drug for the prevention and treatment of PCP is TMPSMX. Studies have shown that the survival rates of HIV-infected patients with PCP are only about 70\% when using TMP-SMZ alone as first- 
line therapy, and survival rates using TMP-SMZ are even lower in moderate to severe cases. Thomas et al. reported an overall survival rate of $93 \%$, and a survival rate of $81 \%$ in patients with severe disease, among $73 \mathrm{HIV}$-infected patients treated with TMP-SMX [18]. Some moderate to severe cases can also prove fatal despite full supportive intensive care with mechanical ventilation. This observation is also supported by the results of our prospective study. In our study, the mortality rate of patients with moderate to severe PCP and HIV was $13.04 \%$ in the TMP-SMX monotherapy group. We observed that the TMP-SMX plus clindamycin group and the TMP-SMX plus caspofungin group showed no further decrease in mortality compared with those prescribed TMP-SMX only.

Previous case reports showed that the efficacy of caspofungin was favorable when it was used as a first-line drug or for salvage therapy for PCP [19]. A trial involving the caspofungin salvage therapy for PCP showed a high success rate (80\%) among HIV-infected patients [20]. In contrast, Kamboj et al. described four HIV-negative patients with PCP that had no observable clinical response to caspofungin as salvage therapy [21]; however, their study is consistent with ours. We found that both therapeutic combinations (TMP-SMX plus clindamycin and TMP-SMX plus caspofungin) demonstrate similar efficacy for the treatment of moderate to severe PCP as TMP-SMX monotherapy. In recent years, the abuse of antibiotics has increased, which not only facilitates the emergence of antibiotic drug resistance but also results in large amounts of wasted antibiotics and of antibiotics entering the water table and waterways, causes an unnecessary economic burden on patients, and causes unnecessary adverse reactions. Currently, China has come to inherit the unfortunate distinction of becoming one of the world's worst abusers of antibiotics [22]. Hence, if TMP-SMX monotherapy can be demonstrated to be as effective and efficient as combinations with other antibiotics and antifungals (as has been demonstrated with the results of our study) for the treatment of moderate to severe PCP, then obviously combinations of TMP/SMX with other antibiotics and antifungals should not be used.

The adverse events of TMP-SMX have been widely reported, and include rashes, fever, gastrointestinal complications, bone marrow suppression, hyperkalemia, hepatoxicity, and renal insufficiency [23]. The adverse effects of clindamycin and caspofungin are similar to those of TMP-SMX. In our study, we there was a high incidence of bone marrow suppression, hepatic dysfunction, and electrolyte disturbances, both at 4 weeks and at 12 weeks, in all three groups, and there were no significant differences in adverse effect rates among the three groups, suggesting that combination therapy with TMPSMX and clindamycin or caspofungin did not increase the incidence of adverse events in subjects compared with TMP-SMX monotherapy. In addition, we failed to identify individual cases of interstitial nephritis, pancreatitis, and aseptic meningitis in our cohort.

Our study had a few limitations. Firstly, not all patients with moderate to severe PCP underwent bronchoscopic examination and bronchoalveolar lavage for definitive PCP diagnosis because hypoxia and dyspnea in some patients made it technically difficult and potentially hazardous to undertake those procedures; nevertheless, we adopted a unified clinical diagnostic standard for patients with moderate to severe PCP who could not undergo those procedures. Secondly, a limited sample size and the non-randomized nature of our study may have introduced a degree of bias into our study. Also, selection and unmeasured confounding bias cannot be completely excluded. Novel therapeutic interventions should ideally be assessed via randomized, controlled clinical trials. However, such an approach may not be practically feasible in the context of patients with critical illness, especially in immunocompromised patients. We endeavored to include patients at a $1: 1: 1$ ratio, so as to reduce inherent bias caused by the subjective intentionality of clinicians in the study. Thirdly, as a result of TMP-SMX drug resistance being rarely reported in China, we did not 
investigate the occurrence of drug resistance in $P$. jirovecii in this study.

\section{CONCLUSION}

Our results indicate that there are no significant differences among the three different treatment regimens in terms of antifungal effectiveness in HIV-infected patients with moderate to severe PCP. Caspofungin and clindamycin are expensive, may not be readily available to patients, and are generally used to manage quite specific infectious agents and diseases, while TMP/SMX is inexpensive, readily available, and is commonly used. TMP-SMX monotherapy as a therapeutic drug regimen to treat HIV-infected patients with moderate to severe PCP is therefore an economically viable, convenient, and appropriate treatment strategy in resource-limited settings.

\section{ACKNOWLEDGEMENTS}

Funding. This work and the journal's Rapid Service Fee was supported by the National Science and Technology Major Project of China During the 13th Five-year Plan Period (2018ZX10302104001), the Medical Research Projects of Chongqing Municipal Science and Technology Bureau and Health Commission (2020GDRC004) and (2020MSXM097), the Chongqing Science and Technology Bureau Project (cstc2020jscx-cylh0004). The funders of the present study had no role in the study design, data collection, data analysis, data interpretation, or writing of the report. The corresponding authors had full access to all the data used in the study, and had final responsibility for the decision to submit for publication.

Medical Writing, Editorial, and Other Assistance. We thank staff of Chongqing Public Health Medical Center for the clinical care given to the enrolled patients, and for facilitating access to relevant medical records. We thank Qing Yang of the First Affiliated Hospital, College of Medicine, Zhejiang University, for supporting this study.

Authorship. All named authors meet the International Committee of Medical Journal Editors (ICMJE) criteria for authorship for this article, take responsibility for the integrity of the work as a whole, and have given their approval for this version to be published.

Author Contributions. YC and JN conceived the study, YQ, JY, ML, JY, XC, YZ, and ZJ collected and collated the data, HC and YL analyzed the data, XH, LX, and GZ interpreted the results, $\mathrm{YH}$ and $\mathrm{XH}$ wrote the manuscript. $\mathrm{VH}$, $\mathrm{YC}, \mathrm{DZ}$, and BZ revised and copy-edited the manuscript. All authors read and approved the final manuscript.

Disclosures. Yinqiu Huang, Xiaoqing He, Hui Chen, Vijay Harypursat, Yanqiu Lu, Jing Yuan, Jingmin Nie, Min Liu, Jianhua Yu, Yulin Zhang, Zhongsheng Jiang, Yingmei Qin, Lijun $\mathrm{Xu}$, Guoqiang Zhou, Defa Zhang, Xiaohong Chen, Baisong Zheng, and Yaokai Chen have nothing to disclose.

Compliance with Ethics Guidelines. Our study received human research ethics approval (Approval Number: 2019-003-02-KY) from the Ethics Committee of the Chongqing Public Health Medical Center, and from the individual institutional ethics committees of each of the other fifteen hospitals involved in this study (Table S1), and was duly registered at the Chinese Clinical Trial Registry (Registration number: ChiCTR1900021195). The study was conducted in accordance with the tenets of the 1964 Declaration of Helsinki. All enrolled patients provided written informed consent.

Data Availability. The datasets generated during and/or analyzed during the present study are not publicly available due to the proprietary nature of the database; however, they are available from the corresponding author upon reasonable request. 
Open Access. This article is licensed under a Creative Commons Attribution-NonCommercial 4.0 International License, which permits any non-commercial use, sharing, adaptation, distribution and reproduction in any medium or format, as long as you give appropriate credit to the original author(s) and the source, provide a link to the Creative Commons licence, and indicate if changes were made. The images or other third party material in this article are included in the article's Creative Commons licence, unless indicated otherwise in a credit line to the material. If material is not included in the article's Creative Commons licence and your intended use is not permitted by statutory regulation or exceeds the permitted use, you will need to obtain permission directly from the copyright holder. To view a copy of this licence, visit http://creativecommons.org/licenses/by$\mathrm{nc} / 4.0 /$.

\section{REFERENCES}

1. Catherinot E, Lanternier F, Bougnoux ME, Lecuit M, Couderc LJ, Lortholary O. Pneumocystis jirovecii pneumonia. Infect Dis Clin N Am. 2010;24(1): 107-38.

2. Walzer PD, Evans HE, Copas AJ, Edwards SG, Grant AD, Miller RF. Early predictors of mortality from Pneumocystis jirovecii pneumonia in HIV-infected patients: 1985-2006. Clin Infect Dis. 2008;46(4): 625-33.

3. Miller RF, Huang L, Walzer PD. Pneumocystis pneumonia associated with human immunodeficiency virus. Clin Chest Med. 2013;34(2):229-41.

4. Yang JJ, Huang CH, Liu CE, et al. Multicenter study of trimethoprim/sulfamethoxazole-related hepatotoxicity: incidence and associated factors among HIV-infected patients treated for Pneumocystis jirovecii pneumonia. PLoS ONE. 2014;9(9):e106141.

5. Cushion MT, Collins MS. Susceptibility of pneumocystis to echinocandins in suspension and biofilm cultures. Antimicrob Agents Chemother. 2011;55(10):4513-8.

6. Roux A, Gonzalez F, Roux M, et al. Update on pulmonary Pneumocystis jirovecii infection in non-HIV patients. Med Mal Infect. 2014;44(5):185-98.
7. Liu Y, Liu Y, Li J, et al. Cappofenjin combined with compound sulfamethoxazole in the treatment of severe pneumocystis pneumonia. Int J Respir. 2009;12:709-12.

8. Castro JG, Morrison-Bryant M. Management of Pneumocystis Jirovecii pneumonia in HIV infected patients: current options, challenges and future directions. HIV AIDS (Auckl). 2010;2:123-34.

9. Guidelines for the Prevention and Treatment of Opportunistic Infections in Adults and Adolescents with HIV (Last updated May 29, 2019; last reviewed June 26, 2019). Recommendations from the Centers for Disease Control and Prevention, the National Institutes of Health, and the HIV Medicine Association of the Infectious Diseases Society of America. https://clinicalinfo.hiv.gov/sites/default/files/guide lines/documents/Adult_OI.pdf.

10. Fu K, Fu YJ, Wang LQ, et al. Meta-analysis of efficacy and safety of compound sulfamethoxazole combined with clindamycin in the treatment of pneumocystis pneumonia. Int J Epidemiol Infect Dis. 2019;05:389-94.

11. Lee WS, Hsueh PR, Hsieh TC, et al. Caspofungin salvage therapy in Pneumocystis jirovecii pneumonia. J Microbiol Immunol Infect. 2017;4:547-8.

12. AIDS Professional Group. AIDS with invasive fungal disease diagnosis and treatment of experts consensus. Chin J Infect Dis. 2019;10:581-93.

13. Zhang G, Chen M, Zhang S, et al. Efficacy of caspofungin combined with trimethoprim/sulfamethoxazole as first-line therapy to treat non-HIV patients with severe pneumocystis pneumonia. Exp Ther Med. 2018;15(2):1594-601.

14. Wang M, Lang G, Chen Y, et al. A pilot study of echinocandin combination with trimethoprim/sulfamethoxazole and clindamycin for the treatment of AIDS patients with pneumocystis pneumonia. J Immunol Res. 2019;2019:1-5.

15. Gingerich AD, Norris KA, Mousa JJ. Pneumocystis pneumonia: immunity, vaccines and treatments. Pathogens. 2021;10(2):236.

16. Jin F, Liu X-H, Chen W-C, Fan Z-L, Wang H-L. High initial $(1,3)$ beta-D-glucan concentration may be a predictor of satisfactory response of caspofungin combined with TMP/SMZ for HIV-negative patients with moderate to severe Pneumocystis jirovecii pneumonia. Int J Infect Dis. 2019;88:141-8.

17. Mu XD, Que CL, He B, Wang GF, Li HC. Caspofungin in salvage treatment of severe pneumocystis pneumonia: case report and literature review. Chin Med J (Engl). 2009;122(8):996-9. 
18. Thomas M, Rupali P, Woodhouse A, Ellis-Pegler R. Good outcome with trimethoprim $10 \mathrm{mg} / \mathrm{kg} / \mathrm{day}-$ sulfamethoxazole $50 \mathrm{mg} / \mathrm{kg} /$ day for Pneumocystis jirovecii pneumonia in HIV infected patients. Scand J Infect Dis. 2009;41(11-12):862-8.

19. Tian Q, Si J, Jiang F, et al. Caspofungin combined with TMP/SMZ as a first-line therapy for moderateto-severe PCP in patients with human immunodeficiency virus infection. HIV Med. 2021;22(4): 307-13.

20. Armstrong-James D, Stebbing J, John L, et al. A trial of caspofungin salvage treatment in PCP pneumonia. Thorax. 2011;66(6):537-8.

21. Kamboj M, Weinstock D, Sepkowitz KA. Progression of Pneumocystis jiroveci pneumonia in patients receiving echinocandin therapy. Clin Infect Dis. 2006;43(9):e92-94.

22. Xu EX, Wu ST, Yuan RX, Zhang CH, Zhang Y. A review of antibiotic abuse research. Modern Trade Ind. $2019 ; 40(36): 72$.

23. Gingerich AD, Norris KA, Mousa JJ. Pneumocystis pneumonia: immunity, vaccines, and treatments. Pathogens. 2021;10(2):236.

\section{Publisher's Note}

Springer Nature remains neutral with regard to jurisdictional claims in published maps and institutional affiliations. 